\title{
A Short Proof of the Minimality of Simons Cone
}

\author{
G. De Philippis (*) - E. Paolini (**)
}

Abstract - In 1969 Bombieri, De Giorgi and Giusti proved that Simons cone is a minimal surface, thus providing the first example of a minimal surface with a singularity. We suggest a simplified proof of the same result. Our proof is based on the use of sub-calibrations, which are unit vector fields extending the normal vector to the surface, and having non-positive divergence. With respect to calibrations (which are divergence free) sub-calibrations are more easy to find and anyway are enough to prove the minimality of the surface.

\section{Introduction.}

The minimality of Simons cone is a very important step in the theory of regularity of minimal surfaces in higher dimension. In 1960s, Reifenberg, De Giorgi and Federer and Fleming, found the first regularity results for minimal surfaces in $\mathbb{R}^{n}$. They found only a partial regularity result, in the sense that minimal surfaces were proven to be regular outside a set (the singular set) of small dimension. Subsequent results of De Giorgi, Fleming, Almgren and Simons gave more precise limitations to the singular set, finally proving that an $(n-1)$-dimensional minimal surface in $\mathbb{R}^{n}$ is regular outside a singular set whose dimension is at most $n-8$.

Simons cone is an example showing that the partial regularity result is optimal: the cone

$$
S=\left\{x \in \mathbb{R}^{8}: x_{1}^{2}+x_{2}^{2}+x_{3}^{2}+x_{4}^{2}=x_{5}^{2}+x_{6}^{2}+x_{7}^{2}+x_{8}^{2}\right\}
$$

is minimal and has a singular point in the origin. In 1969 Bombieri, De Giorgi and Giusti [2] proved that this surface is indeed minimal, thus

(*) Indirizzo dell'A.: via Bellini 7, 50144 Firenze.

E-mail: nemo1685@yahoo.it

(**) Indirizzo dell'A.: Dipartimento di Matematica "U Dini", viale Morgagni 67A, 50134 Firenze.

E-mail: paolini@math.unifi.it 
completing the regularity theory for minimal hyper-surfaces. Since the blow up of a minimal surface in every point is a minimal cone (if the point is non singular, the cone is actually a plane) we understand that the study of minimal cones is very important in the theory of minimal surfaces. Actually this is true not only for the minimizer of the area functional but also for all those geometrical functionals whose principal term is the area.

After the first proof of the minimality of Simons cone, other simplifications and generalizations were obtained. In particular we would like to mention Lawson [6], Simoes [12], Miranda [9] Massari and Miranda [7], Giusti [5], Concus and Miranda [3], Morgan [11], Benarros and Miranda [1], Davini [4].

In this paper, which originates from the Degree Thesis of the first author, we present yet another proof of the minimality of Simons cone.

The original proof of Bombieri, De Giorgi and Giusti and the following simplifications (see [4]) use the nice tool of calibrations. A calibration is a divergence free unit vector field $\xi$ which extends the normal field of the surface to the whole ambient space. Using the divergence theorem one finds that if such a field can be found, then the surface is minimal. Using the symmetries of the cone $S$, the problem of finding a calibration can be reduced to a 2-dimensional problem, and the differential equation $\operatorname{div} \xi=0$ can be reduced to an ODE. Then a somewhat deep study of this equation gives the existence of a global solution with the desired properties.

The proof originally given by Massari and Miranda (see [10] and [8]) uses a different approach. Over the cone $S$ one constructs the cylinder $S \times \mathbb{R} \subseteq \mathbb{R}^{n+1}$. This cylinder can be approximated by the graphs of an increasing sequence of functions $u_{k}: \mathbb{R}^{n} \rightarrow \mathbb{R}$ which are sub-solutions to the minimal surfaces equation $\operatorname{div}\left[D u / \sqrt{1+|D u|^{2}}\right]=0$. Hence the cylinder is a sub-minimal surface. Reasoning in a similar way on the complementary set one finds that the cylinder $S \times \mathbb{R}$ is minimal and hence also the cone $S$ is minimal.

We put together ideas from both these two approaches to find a simplified proof. In fact we consider sub-minimal surfaces i.e. oriented surfaces which are minimal with respect to the internal deformations (Definition 1.1) and we prove a sub-calibration result for the sub-minimal surfaces (Definition 1.4, Theorem 1.5). Hence we explicitly find a sub-calibration for the Simons cone (Section 2) obtaining the sub-minimality of the cone $S$. Passing to the complementary set (i.e. changing the orientation of 
$S$ ) we find that $S$ is also super-minimal and hence it is indeed minimal (Proposition 1.2).

This method simplifies the original approach with calibrations because we are able to explicitly find the sub-calibration (we only need to check the inequality $\operatorname{div} \xi \leq 0$ instead of proving the existence of a solution to the equation). Also this is a simplification with respect to the proof involving sub-solutions to the minimal surface equation because we don't need to pass to higher dimension, to consider the functional on the graphs, and then to go back to the original space.

\section{Sub-minimal sets and sub-calibrations}

In the following we consider, as ambient space, an open set $\Omega \subseteq \mathbb{R}^{n}$. For a measurable set $E \subseteq \mathbb{R}^{n}$ we define the perimeter of $E$ in $\Omega$ as $\left({ }^{1}\right)$.

$$
P(E, \Omega)=\sup \left\{\int_{E} \operatorname{div} g d x: g \in C_{c}^{1}\left(\Omega, \mathbb{R}^{n}\right),|g| \leq 1\right\} .
$$

If $E$ has a regular boundary, $P(E, \Omega)$ is equal to the ( $n-1)$-dimensional surface area of the boundary $\partial E \cap \Omega$.

Definition 1.1. (minimal and subminimal sets). We say that a measurable set $E$ is a local minimum of the perimeter or simply minimal in $\Omega$, if in all bounded open sets $A \subseteq \Omega$ one has $\left({ }^{2}\right)$

$$
P(E, A) \leq P(F, A) \text { for all } F \text { such that } E \triangle F \Subset A \text {. }
$$

We say that $E$ is sub-minimal in $\Omega$, if in all bounded open sets $A \subseteq \Omega$ one has

$$
P(E, A) \leq P(F, A) \quad \text { for all } F \subseteq E \text { such that } E \backslash F \Subset A .
$$

Proposition 1.2. If both $E$ and $E^{c}=\Omega \backslash E$ are sub-minimal in $\Omega$, then $E$ is minimal in $\Omega$.

$\left.{ }^{1}\right)$ We denote with $\mathcal{C}_{c}^{1}(\Omega, \mathbb{R})$ the set of $\mathcal{C}^{1}$ real valued functions with compact support in $\Omega$.

$\left.{ }^{2}\right)$ With $E \triangle F$ we denote the symmetric difference $(E \backslash F) \cup(F \backslash E)$; with $F \Subset E$ we mean that the closure of $F$ is a compact subset of $E$. 
Proof. Let $A$ be a given bounded open subset of $\Omega$ and suppose $E \triangle F \Subset A$. Then consider $F^{\prime}=E \cap F$ and $F^{\prime \prime}=(E \cup F)^{c}$. Clearly $F^{\prime} \subseteq E$ and $F^{\prime \prime} \subseteq E^{c}$. Moreover $E \backslash F^{\prime}=E \backslash F \subseteq E \triangle F \Subset A$ and $E^{c} \backslash F^{\prime \prime}=$ $=E^{c} \backslash F^{c} \subseteq E \triangle F \Subset A$. So, by the sub-minimality of $E$ we get

$$
P(E, A) \leq P\left(F^{\prime}, A\right)=P(E \cap F, A) .
$$

The minimality of $E^{c}$ instead gives $P\left(E^{c}, A\right) \leq P\left(F^{\prime \prime}, A\right)$ which can be written as

$$
P(E, A) \leq P\left(\left(F^{\prime \prime}\right)^{c}, A\right)=P(E \cup F, A) .
$$

From (1) and (2) we get

$$
2 P(E, A) \leq P(E \cap F, A)+P(E \cup F, A)
$$

while, as a general property of perimeter, we know that

$$
P(E \cap F, A)+P(E \cup F, A) \leq P(E, A)+P(F, A) .
$$

Putting together (3) and (4) we conclude

$$
P(E, A) \leq P(F, A)
$$

which gives the minimality of $E$.

Proposition 1.3. (convergence of subminimal sets). Let $E_{k}$ and $E$ be measurable sets in $\Omega$ with $E_{k} \subseteq E$ and suppose that $\left({ }^{3}\right) E_{k} \rightarrow E$ in $L_{\mathrm{loc}}^{1}(\Omega)$. If $E_{k}$ is sub-minimal in $\Omega$ for every $k$, then also $E$ is sub-minimal in $\Omega$.

Proof. Let $A$ be an open bounded subset of $\Omega$ and let $F$ be any measurable set such that $F \subseteq E$ and $E \backslash F \Subset A$. Consider the sets $F_{k}^{\prime}=F \cap E_{k}$. Clearly $F_{k}^{\prime} \subseteq E_{k}$. Moreover $E_{k} \backslash F_{k}^{\prime} \subseteq E \backslash F \Subset A$. So, by the minimality of $E_{k}$ we get

$$
P\left(E_{k}, A\right) \leq P\left(F_{k}^{\prime}, A\right)=P\left(E_{k} \cap F, A\right) .
$$

Notice also that $E_{k} \subseteq E_{k} \cup F \subseteq E$, hence $E_{k} \cup F$ converges to $E$ in $L^{1}$. By the lower-semicontinuity of the perimeter we get

$$
P(E, A) \leq \liminf _{k} P\left(E_{k} \cup F, A\right) .
$$

$\left({ }^{3}\right)$ We say that $E_{k} \rightarrow E$ in $L_{\text {loc }}^{1}(\Omega)$ if for every bounded set $A \subset \Omega$ we have $\left|\left(E_{k} \triangle E\right) \cap A\right| \rightarrow 0$ where $|X|$ is the Lebesgue measure of the set $X$. 
Applying the inequality (4) we obtain

$$
P(E, A) \leq \liminf _{k}\left[P\left(E_{k}, A\right)+P(F, A)-P\left(E_{k} \cap F, A\right)\right]
$$

and by (5) we conclude

$$
P(E, A) \leq P(F, A) .
$$

Hence $E$ is a sub-minimal set.

DEFINITION 1.4. (subcalibration). Let $E \subseteq \Omega$ be a measurable set such that the boundary $\partial E \cap \Omega$ has $\mathcal{C}^{2}$ regularity. We say that a vector field $\xi \in \mathcal{C}^{1}\left(\Omega, \mathbb{R}^{n}\right)$ is a sub-calibration of $E$ in $\Omega$ if it satisfies the following properties: $x \in \partial E \cap \Omega$;

(i) $\xi(x)=v_{E}(x)$ is the exterior normal vector to $\partial E$ for all

(ii) $\operatorname{div} \xi(x) \leq 0$ for all $x \in E \cap \Omega$;

(iii) $|\xi(x)| \leq 1$ for all $x \in \Omega$.

THeOREm 1.5. (subminimality of subcalibrated sets). If $\xi$ is a subcalibration of a set $E$ with boundary of class $\mathcal{C}^{2}$ in $\Omega$ then $E$ is subminimal in $\Omega$.

Proof. Let $A \subseteq \Omega$ be any open, bounded set and let $F \subseteq E$ be a measurable set with $E \backslash F \Subset A$. Choose a sequence of functions $\eta_{j} \in \mathcal{C}_{c}^{1}(A, \mathbb{R})$ such that $\eta_{j}(x)=1$ for $x \in E \backslash F, 0 \leq \eta_{j}(x) \leq 1$ for all $x \in A$ and such that the sequence of sets $A_{j}=\left\{x \in A: \eta_{j}(x)=1\right\}$ is increasing and $\bigcup A_{j}=A$.

If we define $\xi_{j}=\eta_{j} \xi$, we have

$$
\int_{E \cap A} \operatorname{div} \xi_{j}-\int_{F \cap A} \operatorname{div} \xi_{j}=\int_{E \backslash F} \operatorname{div} \xi_{j}=\int_{E \backslash F} \operatorname{div} \xi \leq 0
$$

which means that

$$
\int_{E \cap A} \operatorname{div} \xi_{j} \leq \int_{F \cap A} \operatorname{div} \xi_{j}
$$

Since $\xi_{j} \in \mathcal{C}_{c}^{1}\left(A, \mathbb{R}^{n}\right)$, we have

$$
\int_{F \cap A} \operatorname{div} \xi_{j} \leq \sup \left\{\int_{F \cap A} \operatorname{div} \psi: \psi \in \mathcal{C}_{c}^{1}\left(A, \mathbb{R}^{n}\right),|\psi| \leq 1\right\}=P(F, A) .
$$


On the other hand $\left({ }^{4}\right)$

$$
\begin{aligned}
\int_{E \cap A} \operatorname{div} \xi_{j} & =\int_{\partial E \cap A}\left\langle\xi_{j}, v_{E}\right\rangle d \mathcal{H}^{n-1}=\int_{\partial E \cap A} \eta_{j}\left\langle\xi, v_{E}\right\rangle d \mathcal{H}^{n-1} \\
& =\int_{\partial E \cap A} \eta_{j} d \mathcal{H}^{n-1} \geq \mathcal{H}^{n-1}\left(\partial E \cap A_{j}\right)
\end{aligned}
$$

where $A_{j}=\left\{x \in A: \eta_{j}(x)=1\right\}$. Since we have chosen $\eta_{j}$ so that $A_{j} \uparrow A$, passing to the limit in (8) we obtain

$$
\liminf _{j \rightarrow \infty} \int_{E \cap A} \operatorname{div} \xi_{j} \geq \mathcal{H}^{n-1}(\partial E \cap A)=P(E, A) .
$$

Putting together (6), (7) and (9) we obtain

$$
P(E, A) \leq P(F, A)
$$

which is the sub-minimality of $E$.

\section{Simons cone.}

Let $n=2 m$, and consider the cone

$$
C=\left\{(x, y) \in \mathbb{R}^{m} \times \mathbb{R}^{m}:|x| \leq|y|\right\} \subseteq \mathbb{R}^{n} .
$$

This cone is the zero sub-level $C=\{f \leq 0\}$ of the function $f: \mathbb{R}^{n} \rightarrow \mathbb{R}$

$$
f(x, y)=\frac{|x|^{4}-|y|^{4}}{4}, \quad(x, y) \in \mathbb{R}^{m} \times \mathbb{R}^{m}
$$

hence the following sequences of sets

$$
\begin{aligned}
& E_{k}=\left\{(x, y) \in \mathbb{R}^{m} \times \mathbb{R}^{m}: f(x, y) \leq-\frac{1}{k}\right\}, \\
& F_{k}=\left\{(x, y) \in \mathbb{R}^{m} \times \mathbb{R}^{m}: f(x, y) \leq \frac{1}{k}\right\}
\end{aligned}
$$

both converge to $C$ in $L_{\text {loc }}^{1}\left(\mathbb{R}^{n}\right)$, with $E_{k} \subseteq C$ and $F_{k} \supseteq C$. More precisely, $\left|A \cap\left(C \backslash E_{k}\right)\right| \rightarrow 0$ and $\left|A \cap\left(F_{k} \backslash C\right)\right| \rightarrow 0$ for every bounded set $A$. We are

$\left({ }^{4}\right)$ The Hausdorff measure $\mathcal{H}^{n-1}$ on a regular set corresponds to the $(n-1)$ dimensional surface area of the set. 
going to prove that all the sets $E_{k}$ and $F_{k}^{c}=\mathbb{R}^{n} \backslash F_{k}$ are sub-minimal. To achieve this we will show that the vector field

$$
\xi=\frac{D f}{|D f|}
$$

is a sub-calibration.

Proposition 2.1. If $m \geq 4$, the vector field $\xi$ defined in (10), (11) is a sub-calibration of the sets $E_{k}$ in $\Omega=\mathbb{R}^{n} \backslash\{0\}$ while the vector field - $\xi$ is a sub-calibration of the sets $F_{k}^{c}$ in $\Omega$.

Proof. Clearly $|\xi|=1$ everywhere in $\Omega$. Also we know that the gradient $D f$ is orthogonal to the level sets $\partial E_{k}$ and $\partial F_{k}$, and points outwards the sublevel. So $\xi=D f /|D f|$ is the exterior normal vector to $E_{k}$ in $\partial E_{k}$ and $-\xi$ is the exterior normal vector to $F_{k}^{c}$ in $\partial F_{k}=\partial F_{k}^{c}$.

We want to compute the divergence of $\xi=D f /|D f|$. We recall that

$$
\begin{gathered}
f=\frac{1}{4}\left(|x|^{4}-|y|^{4}\right), \\
f_{x_{i}}=|x|^{2} x_{i}, \quad f_{y_{i}}=-|y|^{2} y_{i}, \quad|D f|^{2}=|x|^{6}+|y|^{6}, \\
f_{x_{i} x_{j}}=2 x_{i} x_{j}+\delta_{i j}|x|^{2}, \quad f_{y_{i} y_{j}}=-2 y_{i} y_{j}-\delta_{i j}|y|^{2}, \quad f_{x_{i} y_{j}}=0 .
\end{gathered}
$$

So

$$
\begin{aligned}
\left(\frac{f_{x_{i}}}{|D f|}\right)_{x_{i}} & =\frac{\left(2 x_{i}^{2}+|x|^{2}\right)\left(|x|^{6}+|y|^{6}\right)-\sum_{j}|x|^{2} x_{i}|x|^{2} x_{j}\left(2 x_{i} x_{j}+\delta_{i j}|x|^{2}\right)}{|D f|^{3}}, \\
\sum_{i}\left(\frac{f_{x_{i}}}{|D f|}\right)_{x_{i}} & =\frac{(2+m)|x|^{2}\left(|x|^{6}+|y|^{6}\right)-2|x|^{8}-|x|^{8}}{|D f|^{3}} \\
& =\frac{(m-1)|x|^{8}+(m+2)|x|^{2}|y|^{6}}{|D f|^{3}} .
\end{aligned}
$$

The derivatives with respect to $y_{i}$ are the same, with signs changed. So we have

$$
\begin{aligned}
|D f|^{3} \operatorname{div} \frac{D f}{|D f|} & =(m-1)|x|^{8}+(m+2)|x|^{2}|y|^{6}-(m-1)|y|^{8}-(m+2)|y|^{2}|x|^{6} \\
& =\left(|x|^{4}-|y|^{4}\right)\left[(m-1)|x|^{4}-(m+2)|x|^{2}|y|^{2}+(m-1)|y|^{4}\right] .
\end{aligned}
$$

We want to prove that the sign of this quantity is the same as the sign of 
$|x|^{4}-|y|^{4}$, so we have only to prove that the expression in square brackets is non-negative. With the substitution $t=|x|^{2} /|y|^{2}$ we obtain the relation

$$
(m-1) t^{2}-(m+2) t+(m-1) \geq 0
$$

which is true for every $t$ if the discriminant $\Delta$ is non-positive, i.e.

$$
\Delta=(m+2)^{2}-4(m-1)^{2}=3 m(4-m) \leq 0
$$

which holds true for $m \geq 4$.

So we have proved that $\operatorname{div} \xi$ has the same sign of $|x|^{4}-|y|^{4}$ in particular $\operatorname{div} \xi \leq 0$ on $C \supseteq E_{k}$ and $\operatorname{div} \xi \geq 0$ on $C^{c} \supseteq F_{k}^{c}$. This was the last condition for checking that $\xi$ is a sub-calibration for every $E_{k}$ and $F_{k}^{c}$ in $\Omega$.

THEOREM 2.2. (minimality of the cone). The cone $C$ is a minimal set in $\mathbb{R}^{n}$.

Proof. Proposition 2.1 ensures that we can apply Theorem 1.5 to obtain that the sets $E_{k}$ and $F_{k}^{c}$ are sub-minimal in $\Omega=\mathbb{R}^{n} \backslash\{0\}$. If $A$ is any open subset of $\mathbb{R}^{n}$ then

$$
P(E, A)=P(E, A \backslash\{0\}) \text { for every measurable set } \mathrm{E}
$$

and this is enough to conclude that $E_{k}$ is sub-minimal in the whole space $\mathbb{R}^{n}$ (notice also that $0 \notin \partial E_{k}$ ). The same reasoning gives the subminimality of $F_{k}^{c}$ in $\mathbb{R}^{n}$.

So we have a sequence of sub-minimal sets $E_{k}$ which converge in $L_{\text {loc }}^{1}$ to the cone $C$. Hence, by Proposition 1.3 we conclude that $C$ is itself subminimal. On the other hand $F_{k}^{c}$ are sub-minimal sets converging to $C^{c}$. Hence also $C^{c}$ is sub-minimal. By Proposition 1.2 we conclude that $C$ is minimal.

\section{REFERENCES}

[1] D. Benarros - M. Miranda, Lawson cones and the Bernstein theorem. Advances in geometric analysis and continuum mechanics (1993), pp. 44-56.

[2] E. Bombieri - E. D. Giorgi - E. Giusti, Minimal cones and the Bernstein problem, Inventiones math., 7 (1969), pp. 243-268.

[3] P. Concus - M. Miranda, Macsyma and minimal surfaces, Proc. of Symposia in Pure Mathematics, by the Amer. Math. Soc., 44 (1986), pp. 163-169.

[4] A. Davini, On calibrations for Lawson's cones, Rend. Sem. Mat. Univ. Padova, 111 (2004), pp. 55-70.

[5] E. Giusti, Minimal Surfaces and Functions of Bounded Variation, volume 80 of Monographs in Mathematics. Birkhäuser, 1984. 
[6] J. H. B. Lawson, The equivariant Plateau problem and interior regularity. Trans. Amer. Math. Soc., 173 (1972), pp. 231-249.

[7] U. Massari - M. Miranda, A remark on minimal cones, Boll. Un. Mat. Ital., 6(2-A) (1983), pp. 231-249.

[8] U. Massari, M. Miranda - M. Miranda Jr. The Bernstein problem in higher dimensions, Boll. Un. Mat. Ital., 2008 (to appear).

[9] M. Miranda, Grafici minimi completi, Ann. Univ. Ferrara, 23 (1977), pp. 269-272.

[10] M. Miranda, Superficie minime e il problema di Plateau, Quaderni di Matematica, Dipartimento di Matematica "De Giorgi", Università degli studi di Lecce, 2006.

[11] F. Morgan, Calibrations and new singularities in area-minimizing surfaces: A survey, Variatonal methods, Proc. Conf. (Paris/Fr. 1988), Prog. nonlinear Differ. Equ. Appl., 4 (1990), pp. 392-342.

[12] P. Simoes, $A$ class of minimal cones in $\mathbb{R}^{n}, n \geq 8$, that minimize area, $\mathrm{PhD}$ thesis, University of California, 1973.

Manoscritto pervenuto in redazione il 10 marzo 2008. 
